\title{
INTEGRAL Spectrometer SPI's GRB detection capabilities *
}

\section{GRBs detected inside SPI's FoV and with the anticoincidence system ACS}

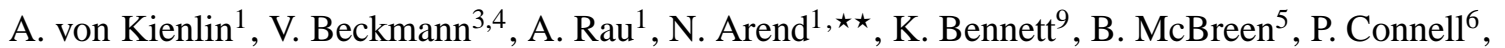 \\ S. Deluit ${ }^{4}$, L. Hanlon ${ }^{5}$, K. Hurley ${ }^{10}$, M. Kippen ${ }^{8}$, G. G. Lichti ${ }^{1}$, L. Moran ${ }^{5}$, R. Preece ${ }^{7}$, J.-P. Roques ${ }^{2}$, \\ V. Schönfelder ${ }^{1}$, G. Skinner ${ }^{2}$, A. Strong ${ }^{1}$, and R. Williams ${ }^{9}$ \\ ${ }^{1}$ Max-Planck-Institut für extraterrestrische Physik, Giessenbachstrasse, 85748 Garching, Germany, \\ 2 Centre d'Études Spatiales des Rayonnements, 9 avenue du Colonel Roche, 31028 Toulouse, France, \\ ${ }^{3}$ Institut für Astronomie und Astrophysik, Universität Tübingen, Sand 1, 72076 Tübingen, Germany \\ ${ }^{4}$ INTEGRAL Science Data Centre, Chemin d'Écogia 16, 1290 Versoix, Switzerland \\ 5 Department of Experimental Physics, University College Dublin, Stillorgan Road, Dublin 4, Ireland \\ 6 Space Research Group, Univ. of Birmingham B15 2TT, Birmingham, UK \\ 7 Dept. of Physics, UAH, National Space Science \& Technology Center, Huntsville, AL 35805, USA \\ 8 Space and Remote Sensing Sciences, NIS-2 Mail Stop D436, Los Alamos National Laboratory, Los Alamos, \\ NM 87545, USA \\ 9 Science Operations and Data Systems Division of ESA/ESTEC, SCI-SDG, Keplerlaan 1, Postbox 299, 2200 AG Noordwijk, \\ The Netherlands \\ ${ }^{10}$ UC Berkeley Space Sciences Laboratory, Berkeley, CA 94720-7450, USA
}

Received 16 July 2003 / Accepted 8 August 2003

\begin{abstract}
The spectrometer SPI, one of the two main instruments of the INTEGRAL spacecraft, offers significant gammaray burst detection capabilities. In its $35^{\circ}$ (full width) field of view SPI is able to localise gamma-ray bursts at a mean rate of $\sim 0.8 /$ month. With its large anticoincidence shield of $512 \mathrm{~kg}$ of BGO crystals SPI is able to detect gamma-ray bursts quasi omni-directionally with a very high sensitivity. Burst alerts of the anticoincidence shield are distributed by the INTEGRAL Burst Alert System. In the first 8 months of the mission about 0.8/day gamma-ray burst candidates and 0.3/day gamma-ray burst positions were obtained with the anticoincidence shield by interplanetary network triangulations with other spacecrafts.
\end{abstract}

Key words. gamma-ray bursts - GRB - gamma-ray astronomy - INTEGRAL - SPI

\section{Introduction}

The detection and investigation of cosmic gamma-ray bursts (GRBs) is an important scientific topic of the INTEGRAL mission. Even now, 35 years after their discovery by the Vela satellites (Klebesadel et al. 1973), this phenomenon is far from being well-understood. A major breakthrough in this field was obtained in the 1990's with the BATSE detectors (Fishman et al. 1989) on NASA's Compton GammaRay Observatory (CGRO). In the ten years of the CGRO

Send offprint requests to: A. von Kienlin,

e-mail: azk@mpe.mpg.de

* Based on observations with INTEGRAL, an ESA project with instruments and science data centre funded by ESA member states (especially the PI countries: Denmark, France, Germany, Italy, Switzerland, Spain), Czech Republic and Poland, and with the participation of Russia and the USA.

$\star \star$ Present address: TU München, Department of Physics, Institute E21, James-Franck-Str., 85748 Garching, Germany. mission 2704 bursts were registered, which showed an isotropic distribution over the entire sky, but with a deficiency for weak burst compared to a homogeneously distribution $(\log N-\log S$ distribution with $-3 / 2$ power law). It was the Italian/Dutch satellite BeppoSAX (Boella et al. 1997) which helped to reveal the cosmological nature of GRBs with the identification of the first X-ray afterglow (Costa et al. 1997), which triggered the first successful follow-up observation at optical wavelengths (van Paradijs et al. 1997) and subsequent direct redshift measurements (Metzger et al. 1997). The redshifts obtained to date for about 30 GRBs range from 0.0085 to 4.5. Observational evidence now strongly suggests that GRBs longer than $2 \mathrm{~s}$ are associated with hypernovae (Galama et al. 1998; Stanek et al. 2003; Hjorth et al. 2003). Gamma-ray polarimetry of the intense event GRB 021206 using the RHESSI satellite (Coburn \& Boggs 2003) indicates that the GRB emission is highly polarised (80\%), suggestive of synchrotron radiation from particles in a highly-ordered magnetic field (Granot 2003). 


\section{GRB detection with the camera of SPI}

The aim of the spectrometer SPI is to perform high-resolution spectroscopy of astrophysical sources in the energy range between $20 \mathrm{keV}$ and $8 \mathrm{MeV}$ (Vedrenne et al. 2003). The imaging capability is good, but is exceeded by that of the imager IBIS which complements SPI by having higher imaging resolution, but lower spectroscopic resolving power (Ubertini et al. 2003). Hence we expect the two main INTEGRAL instruments to make contributions to GRB research in different areas. The connection between GRBs and type Ib/c "hypernovae" (Hjorth et al. 2003) heralds a potentially exciting era for highresolution $\gamma$-ray spectroscopy of the closest of these sources. In addition, the broad energy coverage of SPI is well suited to constrain the spectral shape, both below and above the energy at which the GRB power output is typically peaked ( 250 keV). The long-standing controversy over the existence of short-lived spectral features in GRB spectra can also be addressed by SPI's superb spectroscopic capabilities. On the other hand, IBIS will provide precise GRB locations to the community, which is important for the observation of GRB afterglows. In addition, the capability to cross-calibrate both spectra and images between the two experiments is extremely important, particularly in the case of such short-lived events as GRBs, which cannot be re-observed. Currently GRBs which occur inside SPI's field of view (FoV) are detected and analysed offline. The implementation of an automatic GRB detection algorithm for SPI (von Kienlin et al. 2003a) into the INTEGRAL burst-alert system (IBAS, Mereghetti et al. 2003a) is planned.

\subsection{Imaging with the spectrometer SPI}

The camera of SPI, which consists of 19 cooled high-purity germanium detectors, residing in a cryostat, is shielded on the side walls and rear side by a large anticoincidence shield (ACS). The FoV of the camera is defined by the upper opening of the ACS. The imaging capability of the instrument is attained by a passive-coded mask mounted $1.7 \mathrm{~m}$ above the camera. By observing a series of nearby pointing directions around the source ("dithering") the imaging capability of SPI is improved by reducing ambiguity effects. For the normal mode of operation two dithering patterns are applied, a square one with $5 \times 5$ pointings and a hexagonal one with 7 pointings. Each pointing lasts about $30 \mathrm{~min}$. The slew between pointings on a dither pattern lasts as 5-10 min. Due to their short duration, dithering will not improve the imaging of SPI for bursts. But during its short duration a burst will be in most cases the brightest $\gamma$-ray source for SPI in the whole FoV, so imaging with SPI is still possible. The case when a burst occurred during a slew can be investigated for GRB 030131. Despite the modest angular resolution of SPI, which is of the order of $2.5^{\circ}$, it is possible to locate the direction of bursts down to a few arcminutes.

\subsection{Analysis methods for GRBs}

The analysis of GRBs in the SPI FoV has been performed by using the instrument-specific software, which has been developed by the SPI instrument team in collaboration with the ISDC (Beckmann 2002). In both, image and spectral extraction, the analysis methods are similar and are therefore described here together. SPI is a background-dominated instrument. The overall count rate of the detector plane when observing an empty field is about 880 counts/s, while e.g. the Crab shows a SPI count rate of 33 counts/s. Therefore a careful background handling is essential. In the case of short events as GRBs, the best way to remove the background is to take the observation which encloses the GRB (excluding the time when the GRB was detectable in SPI) as a background. By applying the same parameters as for the GRB analysis a detector spectrum should be extracted. This detector spectrum does not only contain the background, but will also include sources in the FoV and therefore allows an optimised data reduction. The data for the GRB should be extracted from a short time range around the peak, in order to achieve a good signal-tonoise ratio for the result. Source location and spectral extraction are performed applying the iterative-removal-of-sources method, which is implemented in the SPIROS analysis software (Skinner \& Connell 2003). For source location no a priori knowledge of the GRB position is necessary, while in order to extract a spectrum, the best known (e.g. ISGRI derived) source position should be applied.

\subsection{Results obtained for the first bursts observed inside SPI's FoV}

Since the start of the mission, six GRBs have been observed within SPI's FoV. Table 1 summarises the important quantities derived with SPI. In all cases the GRB was detected by IBIS and for comparison the important parameters found from the IBIS data are also shown. Below for each of the GRBs a short summary is given, especially with the emphasis on the SPI performance:

GRB 021125: The first GRB observed within the FoV of INTEGRAL (in this case the partially-coded FoV) was the one with the brightest fluence of the six GRBs in Table 1 (Malaguti et al. 2003). The GRB occurred during a period of the Cyg X-1 in-orbit calibration where SPI was set into a restricted telemetry mode. This was the time period allocated for the IBISPICsIT photon-by-photon mode calibration, which caused high telemetry loads. Only the telemetry, containing the technicaland science-housekeeping data and the onboard spectra, with an aquisition time of $\sim 30 \mathrm{~min}$, were downlinked. Nevertheless it was possible to determin a GRB-lightcurve and -location by using the counter data of the 19 Ge-detectors of SPI's camera, which are sensitive in the broad energy range from $\sim 20 \mathrm{keV}$ to $\sim 8 \mathrm{MeV}$. Due to the high S/N-ratio, with which the GRB was seen in these data, the radius of the obtained error circle is approximately twice the one of IBIS/PICsIT. The error circles of IBIS \& SPI are overlaping each other, thus confirming the reported GRB location. By comparing the count rate of the GRB event with the one of a Crab observation, recorded at a similar off-axis angle $\left(6.45^{\circ}\right)$, one gets the flux and fluence in the $20 \mathrm{keV}-8 \mathrm{MeV}$ range in Crab units. For IBIS it was only possible to extract the mean flux in praticable energy ranges of 
Table 1. Table of GBRs detected inside the FoV of SPI. For comparison the results of IBIS are listed too. The data sets are taken from Malaguti et al. (2003) for GRB 021125, from Mereghetti et al. (2003d) for GRB 021219, from Götz et al. (2003b) for GRB 030131, from Mereghetti et al. (2003c) for GRB 030227, from von Kienlin et al. (2003b) for GRB 030320 and from Beckmann et al. (2003) for GRB 030501. The first column lists the date of the GRB, the second the duration of the burst. Column "GRB Location" quotes for SPI and IBIS-ISGRI (and IBISPICsIT in one case) the centre (in RA and Dec) and radius $r$ of the error circle (90\% confidence value) together with the signal-to-noise (S/N) ratios, with which the GRB was detected. The column "GRB Loc. Offset" lists the the offset angles of centre of the SPI-error circle (SPI) to the one obtained by IBIS and the offset angle of the GRB (IBIS localisation) with respect to the INTEGRAL pointing direction (Point.). The next column specifies the peak flux in the $20-200 \mathrm{keV}$ energy range. The GRB fluence is given for the same energy range. The column "Photon Index" gives the result of a model fit of the GRB spectrum with a power law. Several fields of the table are left blank, because the corresponding values are not reported in the references mentioned above. The fluxes and fluences in Crab units were determined in other energy ranges than the standard $20-200 \mathrm{keV}$ range used for the table (see text).

\begin{tabular}{|c|c|c|c|c|c|c|c|c|c|c|c|}
\hline \multirow[b]{2}{*}{ GRB } & \multirow[b]{2}{*}{ Durat. } & \multirow[b]{2}{*}{ Instr. } & \multicolumn{4}{|c|}{ GRB Location } & \multicolumn{2}{|c|}{ GRB Loc. Offset } & Peak Flux & Fluence & \multirow{2}{*}{$\begin{array}{l}\text { Photon } \\
\text { Index }\end{array}$} \\
\hline & & & RA (J2000) & Dec (J2000) & $r$ & $\mathrm{~S} / \mathrm{N}$ & SPI & Point. & $\frac{\text { photons }}{\mathrm{cm}^{2} \mathrm{~s}}$ & {$\left[\frac{\mathrm{erg}}{\mathrm{cm}^{2}} \times 10^{-6}\right]$} & \\
\hline \multirow{2}{*}{030501} & \multirow{2}{*}{$\sim 40 \mathrm{~s}$} & SPI & $19^{\mathrm{h}} 07^{\mathrm{m}}$ & $06^{\circ} 25^{\prime}$ & $21^{\prime}$ & 15.4 & \multirow{2}{*}{$19^{\prime}$} & \multirow{2}{*}{$13.25^{\circ}$} & $3.22 \pm 0.50$ & $3.93 \pm 0.27$ & $1.88 \pm 0.10$ \\
\hline & & ISGRI & $19^{\mathrm{h}} 05^{\mathrm{m}} 30^{\mathrm{s}}$ & $06^{\circ} 18^{\prime} 26^{\prime \prime}$ & $3^{\prime}$ & 10.0 & & & $2.7 \pm 1.0$ & $3 \pm 1$ & $1.75 \pm 0.10$ \\
\hline \multirow[b]{2}{*}{030320} & \multirow[b]{2}{*}{$\sim 60 \mathrm{~s}$} & SPI & $17^{\mathrm{h}} 53.4^{\mathrm{m}}$ & $-26^{\circ} 2.8^{\prime}$ & $33^{\prime}$ & 7.2 & \multirow{2}{*}{$49.8^{\prime}$} & \multirow{2}{*}{$15.5^{\circ}$} & 3.1 & $13.5_{-2.6}^{+2.1}$ & $1.51 \pm 0.16$ \\
\hline & & ISGRI & $17^{\mathrm{h}} 51^{\mathrm{m}} 42^{\mathrm{s}}$ & $-25^{\circ} 18^{\prime} 44^{\prime \prime}$ & $3^{\prime}$ & 15 & & & 5.7 & 11 & $1.69_{-0.08}^{+0.07}$ \\
\hline \multirow{2}{*}{030227} & \multirow{2}{*}{$\sim 20 \mathrm{~s}$} & $\overline{\text { SPI }}$ & $\bar{~} 4^{\mathrm{h}} 59.1^{\mathrm{m}}$ & $=+20^{\circ} 31.9^{\prime}$ & $31^{\prime}$ & 7.7 & \multirow{2}{*}{$21.6^{\prime}$} & \multirow{2}{*}{$8.55^{\circ}$} & $1.317 \pm 0.270$ & $0.96 \pm 0.14$ & $2.2_{-0.33}^{0.45}$ \\
\hline & & ISGRI & $4^{\mathrm{h}} 57^{\mathrm{m}} 32.2^{\mathrm{s}}$ & $+20^{\circ} 29^{\prime} 54^{\prime \prime}$ & $3^{\prime}$ & & & & 1.1 & 0.75 & $1.85 \pm 0.2$ \\
\hline \multirow{2}{*}{030131} & \multirow{2}{*}{$\sim 150 \mathrm{~s}$} & $\begin{array}{l}\text { SPI } \\
\end{array}$ & & - & & - & \multirow{2}{*}{$0^{\prime}$} & \multirow{2}{*}{$\begin{array}{l}8.6^{\circ}- \\
10.6^{\circ}\end{array}$} & 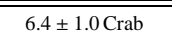 & $137 \pm 8 \mathrm{Crab} \cdot \mathrm{s}$ & - \\
\hline & & ISGRI & $13^{\mathrm{h}} 28^{\mathrm{m}} 21^{\mathrm{s}}$ & $+30^{\circ} 40^{\prime} 33^{\prime \prime}$ & $2.5^{\prime}$ & & & & $\sim 1.9(6.5 \mathrm{Crab})$ & 7 & \\
\hline \multirow{2}{*}{021219} & \multirow{2}{*}{$\sim 6 \mathrm{~s}$} & SPI & $18^{\mathrm{h}} 49.3^{\mathrm{m}}$ & $31^{\circ} 46.1^{\prime}$ & $28^{\prime}$ & 8.5 & \multirow{2}{*}{$17.6^{\prime}$} & \multirow{2}{*}{$9.96^{\circ}$} & $10.7 \pm 1.1 \mathrm{Crab}$ & $30 \pm 3 \mathrm{Crab} \cdot \mathrm{s}$ & - \\
\hline & & ISGRI & $18^{\mathrm{h}} 50^{\mathrm{m}} 25^{\mathrm{s}}$ & $+31^{\circ} 56^{\prime} 23^{\prime \prime}$ & $2^{\prime}$ & 15.5 & & & $\sim 3.7$ & 0.9 & $2.0 \pm 0.1$ \\
\hline \multirow{3}{*}{021125} & \multirow{3}{*}{$\sim 24 \mathrm{~s}$} & SPI & $19^{\mathrm{h}} 47^{\mathrm{m}} 55^{\mathrm{s}}$ & $+28^{\circ} 23^{\prime} 49^{\prime \prime}$ & $13^{\prime}$ & 53 & \multirow{2}{*}{$0.5^{\prime}$} & \multirow{2}{*}{$7.2^{\circ}$} & $22.9 \pm 1.3 \mathrm{Crab}$ & $386 \pm 44 \mathrm{Crab} \cdot \mathrm{s}$ & - \\
\hline & & ISGRI & $19^{\mathrm{h}} 47^{\mathrm{m}} 56^{\mathrm{s}}$ & $+28^{\circ} 23^{\prime} 28^{\prime \prime}$ & $2^{\prime}$ & & & & $14 \pm 2$ Crab (mean) & \multirow{2}{*}{$\begin{array}{l}\sim 51 \text { (in } 20-500 \mathrm{keV} \\
\text { range) }\end{array}$} & $\sim 2.2$ \\
\hline & & PICsIT & $19^{\mathrm{h}} 47^{\mathrm{m}} 51^{\mathrm{s}}$ & $+28^{\circ} 19^{\prime} 16^{\prime \prime}$ & $5^{\prime}$ & & - & - & $9 \pm 1$ Crab (mean) & & $\sim 3.7$ \\
\hline
\end{tabular}

the instruments (ISGRI: $20-180 \mathrm{keV}$, PICsIT: $180-500 \mathrm{keV}$ ). The mean flux of SPI is $9.2 \pm 1.1 \mathrm{Crab}$ and agrees reasonably with the values in Table 1 . The attempt to extract a GRB spectrum from the SPI on-board spectra failed, because there is still an uncertainty in the determination of the time interval, during which the spectrum was recorded.

GRB 021219: Also the second FoV-GRB occurred during the payload performance and verification phase (Mereghetti et al. 2003d). During this time SPI was still in a restricted telemetry mode, due to the telemetry limitations in the beginning of the mission. In this case only the multiple detector events and the events which were processed by the PulseShape-Discriminator (PSD) were downlinked together with the technical- and science-housekeeping data. This mode is especially unsuitable for the analysis of GRBs, because the single events, carrying the energy information below $200 \mathrm{keV}$ are disregarded on board. Thus the same analysis method was used as described for GRB 021125. The GRB location, lightcurve, fluxes and fluence were determined with the help of the counter data of the 19 Ge-detectors. The obtained error circle overlaps with the position reported by ISGRI. The flux and fluence, again in Crab units, agrees with the one determined for ISGRI.

GRB 030131: As the burst occurred during the slew of INTEGRAL, the standard software could not be used to extract the burst as described in Sect. 2.2. As SPI was in a restricted telemetry mode, only multiple events and those single events analysed by the PSD are availabale for the analysis of this burst. Due to the weakness of the burst which adds to the problems of analysing the burst, no reliable position could be derived from the SPI data.

But using again the counter data of the 19 Ge-detectors contained in the science-housekeeping data, it was possible to

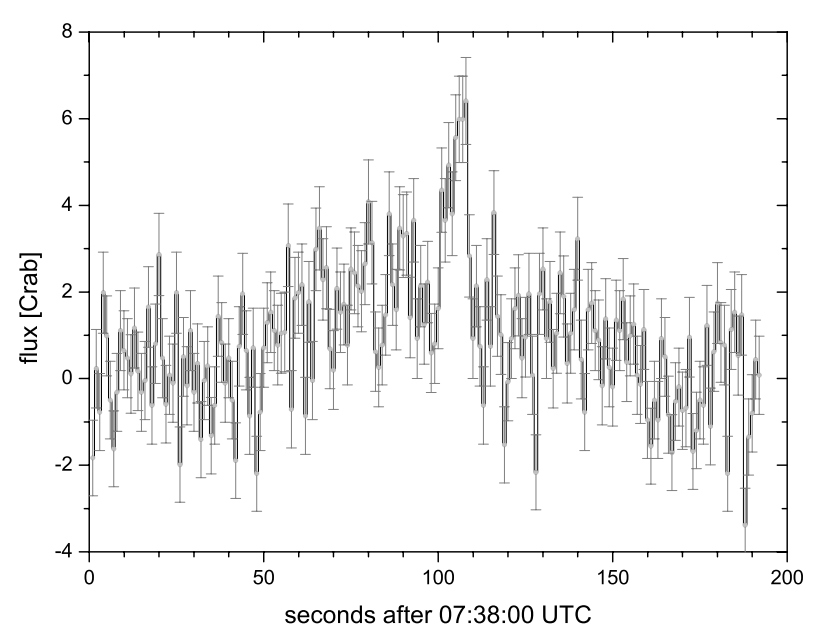

Fig. 1. SPI lightcurve of GRB 030131 obtained from the detector count rates in the energy range $0.02-8 \mathrm{MeV}$, which are part of the scientific-housekeeping data; time starts from 07:38:00 UT. The flux in Crab units has been derived by comparing the count rate with the one measured for the Crab nebula.

determin the lightcurve shown in Fig. 1. The burst can bee seen as an increase of the flux starting at $\sim 07: 38: 50$ UTC until the end of the most prominent peak at 07:39:52 UTC. Probable a part of the next $40 \mathrm{~s}$ can be also attributed to the burst event. Although the spacecraft startet slewing at 07:39:06 the derived lightcurve is not affected. The peak flux at 10:39:48 UTC agrees well with the one reported by ISGRI (Götz et al. 2003b). The fluence listed in Table 1 was determined for the time period 07:38:50-07:39:52 UTC.

GRB 030227: This long (20 s) GRB was the first one which was observed in the SPI field of view, while SPI was in full 
operational mode. It was detected by the IBAS program based on the analysis of the IBIS/ISGRI data (Götz et al. 2003a). Analysis of SPI data on this GRB gave a position $0.36^{\circ}$ off the IBIS position. Even though the burst was weak (total fluence for SPI $\left.F=(9.6 \pm 1.4) \times 10^{-7} \mathrm{ergs} \mathrm{cm}^{-2}\right)$ a spectral extraction in $2 \mathrm{~s}$ wide bins was possible and the SPI data show an indication for hard-to-soft evolution (Mereghetti et al. 2003c). The overall spectrum has a rather steep slope: $\Gamma=2.2_{-0.33}^{0.45}(90 \%$ confidence value).

GRB 030320: So far, this is the GRB observed at the largest off-axis angle, near to edge of the field of view of SPI (2003b). Using the single and multiple detector events SPI was able to localise the GRB in the $100 \mathrm{keV}$ to $1 \mathrm{MeV}$ energy range to an error circle with a radius of 53' (99\% confidence value) which agrees with the IBIS position. The creation of a lightcurve with a $1 \mathrm{~s}$ time binning was only possible when using the Gedetector count rates of the science housekeeping data. The lightcurve exhibits two prominent peaks during the $\sim 60 \mathrm{~s}$ of prompt emission which are separated by $\sim 35 \mathrm{~s}$. The flux, fluence and the photon index for SPI was derived by analysing the count spectrum with an alpha-test version of XSPEC 12.0. It crops up that in the case of this GRB the XSPEC 12.0 derived results are in better agreement, compared to the results obtained with the method described in chapter 2.2. One has to bear in mind that the instrument response for sources observed at such large off-axis angles is not fully understood, because of deficiencies in the knowledge of the mass model.

The hard-to-soft spectral evolution observed for ISGRI is not apparent in the SPI data, nevertheless it was possible to confirm the derived ISGRI fluxes of the first and second peak of emission.

GRB 030501: This long ( 40s) GRB was detected in the partially-coded FoV of both, IBIS and SPI (Mereghetti et al. 2003b). The position and error circle extracted from the SPI data agree with the one obtained by ISGRI. The spectral extraction from both instruments show a similar peak flux, fluence, and spectral shape $(\Gamma=1.8 \pm 0.1$ for a single power law). Also comparison with Ulysses (Hurley et al. 2003b) and RHESSI (Lin et al. 2002) data showed remarkably consistency. Although the flux seems to be correlated with the hardness of the GRB spectrum, there is no clear soft-to-hard evolution seen over the duration of the burst (Beckmann et al. 2003).

\subsection{Discussion of the obtained results}

The first six GRBs observed with INTEGRAL showed that SPI was always able to detect the same event and to confirm in most cases the results obtained with IBIS. For the three first bursts it has to be considered that the capabilites of SPI were weakened by the telemetry limitations in the beginning of the mission. The next event was the weak GRB 030227 and the last two were observed at a large offset angle, where only 3 to 5 of the 19 Ge-detectors were irradiated by the GRB. So the demonstration of SPI's full capabilities in the case of a strong event in the fully-coded FoV still has to take place.
Looking at the current data set summarised in Table 1 most of the GRBs were detected with a S/N between 7 and 16 , with the exception of GRB 021125. At this level SPI is able to localise the GRB down to error radii of $20^{\prime}-30^{\prime}$ and the error circle ( $90 \%$ confidence) overlapped in most cases with the one of IBIS. Also the peak flux, fluence and photon indices agreed approximately with the one derived by IBIS.

The GRB detection rate in the FoV of SPI of $\sim 1 \mathrm{GRB} /$ month in the first six months of the mission and the obtained positioning errors confirmed the simulations and calculations carried out before by Skinner et al. (1997).

\section{GRB detection with ACS}

\subsection{Detection capabilities}

The ACS provides a large effective area for the detection of bursts (von Kienlin et al. 2003a), but unfortunately with no or only very coarse positional information. The ACS consists of 91 BGO crystals with a total mass of $512 \mathrm{~kg}$. The energy range for burst detection is determined by the setting of the energydiscriminators for veto generation, which is a tradeoff between background reduction and dead-time for the SPI camera. As a veto shield the ACS has no upper limit of the energy range. The sensitivity to $\gamma$-rays depends among other things (e.g. projection area) on the attenuation length in the BGO crystals which have thicknesses ranging from $16 \mathrm{~mm}$ at the top to $50 \mathrm{~mm}$ at the bottom near to the Ge-camera.

It is not possible to quote an exact lower energy threshold, due to the redundancy concept chosen for the ACS hardware. Each of the 90 BGO crystals is viewed by two photomultipliers (PMTs), which are read out by 90 front-end electronic (FEE) boxes (The 91th BGO crystal is read out by a single PMT and FEE). The redundancy concept of the ACS is obtained by the cross wiring of neighbouring crystal and electronic boxes. In this way always anode signals from two PMTs connected to neighbouring crystals are summed in one FEE. It emerges that a disadvantage of this method is an uncertainty in the energythreshold value of individual FEEs, caused by different light yields of neighbouring BGO-crystals and different PMT properties like quantum efficiency and amplification. A result of this is that the threshold extends over a wide energy range and is not at all sharp. Since the commissioning phase which took place in the first two months after launch the energy discriminators settings were set at a constant level which corresponds to approximately $75_{-25}^{+75} \mathrm{keV}$.

For the GRB detection on ground with IBAS the ACS housekeeping (HK) data are monitored. These data include the values of the overall veto counter of the veto control unit (VCU) and the individual ratemeter values of each FEE. Both kinds of HK data are suitable for burst detection. The count rate of the overall veto counter (ORed veto signals of all 91 FEEs) is sampled every $50 \mathrm{~ms}$. A packet, containing 160 consecutive count rates, is transmitted every $8 \mathrm{~s}$ to ground. If no gap in the telemetry stream occurs one could have a continuous ACS veto-rate light curve with $50 \mathrm{~ms}$ binning. The measurement time of the individual FEE ratemeter can be adjusted between 0.1 and $2 \mathrm{~s}$. An integration time of $1.048 \mathrm{~s}$ has 
been selected for the mission. All 91 FEEs are read out successively in groups of 8 FEEs every $8 \mathrm{~s}$. The readout of all 91 ratemeter values thus needs $96 \mathrm{~s}$. In contrast to the VCU overall veto counter the individual ratemeter values do not yield a continuous stream. Additionally the values of different FEE groups are shifted by a time interval of $8 \mathrm{~s}$. So it is very difficult, to derive the burst-arrival direction from these individual counting rates.

The SPI/ACS Burst-Alert System is one branch of IBAS. The trigger algorithm used looks for a significant excess with respect to a running average, comparable to the trigger algorithm used for other spacecrafts (e.g. Ulysses). All IBAS processes are multi-threaded applications and run as daemon processes. In the current configuration the ACS overall vetocounter values are monitored simultaneously at 8 different timescales $(0.05 \mathrm{~s}, 0.1 \mathrm{~s}, 0.2 \mathrm{~s}, 0.4 \mathrm{~s}, 0.8 \mathrm{~s}, 1 \mathrm{~s}, 2 \mathrm{~s}$ and $5 \mathrm{~s})$. An alert is generated if two of the monitor programs detect a countrate excess above a predefined significance level, which can be set individually for each timescale. The monitoring of the ratemeter values of individual FEEs is not yet implemented into the ACS part of IBAS but could help to give a rough estimation of the GRB arrival direction. This information could be used to distinguish between the two arrival-cone intersections of the interplanetary network. The SPI-ACS burst alerts distributed by IBAS are providing the time of occurrence in UTC, the spacecraft orbital position (in RA, Dec and distance to geocenter) and a $105 \mathrm{~s}$ light-curve $(5 \mathrm{~s}$ pre burst trigger, $100 \mathrm{~s}$ after burst trigger) with $50 \mathrm{~ms}$ resolution.

Since December 2002 ACS has been added to the 3rd interplanetary network $^{1}$ (IPN) of $\gamma$-ray detectors. During the first year of the INTEGRAL mission the IPN will consist Ulysses, Mars Odyssey 2001, Konus-WIND, HETE-II, RHESSI and of course INTEGRAL/SPI-ACS (Hurley 1997). The network will have an excellent configuration during this time, due to the large spacecraft separations between Earth, Mars and Ulysses, which is orbiting around the sun, out of the ecliptic plane.

\subsection{Results obtained for the first bursts observed with SPI/ACS}

We present here the analysis of GRBs detected with the SPI-ACS during the first eight months (November 2002June 2003) of the INTEGRAL mission. Subsequently, the selection criteria are defined and the burst sample will shortly be discussed.

\subsubsection{Sample selection}

GRBs show a large variety in appearance, e.g. light-curve shape and observer-frame duration. In order to allow a decent statistical analysis, an applicable and robust selection of the sample of GRB events in the SPI-ACS overall rate is required.

Naturally, the best criterion for selecting GRBs from the variety of events in SPI-ACS is the localisation on the sky. As SPI-ACS has no spatial resolution, this selection is fully dependent on the localisation or confirmation of the respective events

\footnotetext{
1 3rd IPN website: http://ssl. berkeley.edu/ipn3/ index.html
}

by other missions (e.g. IPN, HETE-II). These bursts constitute only a subsample of the GRBs detected by SPI-ACS as different instrumental properties of the according missions (e.g. energy range, sensitivity) do not allow the simultaneous observations/detections of all GRBs of the entire sample. Obviously, it is worthwhile to study also the sample of bursts which are only visible in the SPI-ACS rates and not confirmed elsewhere. Probing the very high energies with the unprecedented sensitivity of the SPI-ACS might open new insights into the burst populations and burst physics.

We define our sample based on the only property measurable by the SPI-ACS, the observer-frame light curve. In Fig. 2 three example light curves are shown, representing the known variety of burst shapes (Fishman \& Meegan 1995). The selection criteria for our sample are as follows: an event is categorised as a probable GRB when the total significance above the background exceeds a significance level of $\sigma=12$ in at least one time interval during the event. E.g., assuming a typical background value of 3700 counts per $50 \mathrm{~ms}$ and taking into account the measured FWHM of the ACS background distribution which is larger then the expected poissonion distribution by a factor of 1.6 , e.g. a single $50 \mathrm{~ms}$ event with 4870 total counts (source+background), a $3 \times 50 \mathrm{~ms}$ event with 4500 , 4400 and 4250 counts, respectively, will match the selection. Each event is subsequently checked for solar or particle origin using JEM-X and the GOES web page ${ }^{2}$ and IREM, respectively. Nevertheless, one has to be aware of the only approximatively known (due to varying background) biasing against faint short bursts. Thus, the SPI-ACS GRB sample described here certainly favours the population of long bright bursts. The complete SPI-ACS GRB sample including statistics is available online $\mathrm{e}^{3,4}$.

\subsubsection{Analysis and discussion}

According to our sample selection described above, a total of 145 GRB candidates were detected during the first 8 months of the mission. 58 of these have been confirmed by other instruments. Using the elapsed mission time, we find an approximate rate of GRBs detected by the SPI-ACS of $\sim 290$ ( $\sim 116$ confirmed) per year which is in good agreement with the predictions given in Lichti et al. (2000) prior to the start of the mission. The total rate is comparable also to BATSE (Paciesas et al. 1999).

In addition to the number of events, the SPI-ACS overall rate provides the possibility of deriving the burst duration in the instrumental observer frame and the variability of the light curve. As no spectral resolution exists, typical burst parameters such as fluence and peak flux can not be derived. Only the total integrated counts and the counts in the burst maximum can be extracted from the light curve. In the following, only the distribution of the instrumental observer-frame burst duration will

\footnotetext{
2 http://www.sec.noaa.gov

3 http://www.mpe.mpg.de/gamma/instruments/ integral/spi/acs/grb

4 http://isdc.unige.ch/index.cgi?Science+grb
} 

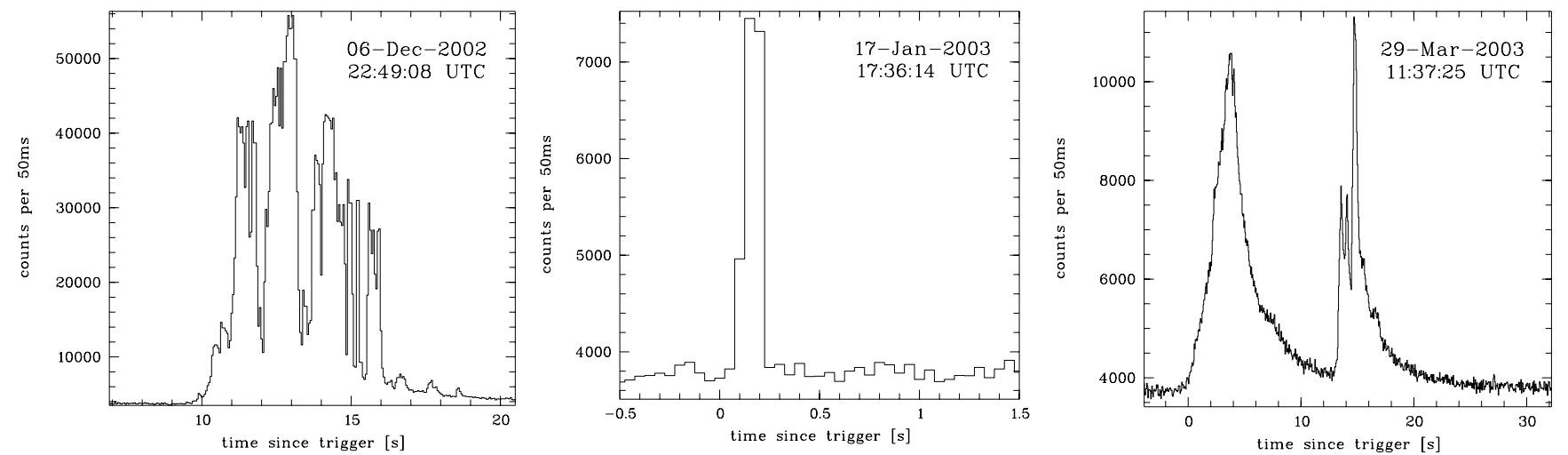

Fig. 2. Overall rate light curves for three typical GRBs detected by SPI-ACS with $50 \mathrm{~ms}$ time resolution. We show (from left to right) the extraordinary bright IPN triangulated burst 021206 (Hurley et al. 2003c), the short triangulated event from 17th of January 2003 (Hurley et al. $2003 \mathrm{a}$ ) and the extensively studied (Stanek et al. 2003; Hjorth et al. 2003 and references therein), nearby ( $z=0.168$; Greiner et al. 2003) GRB 030329.

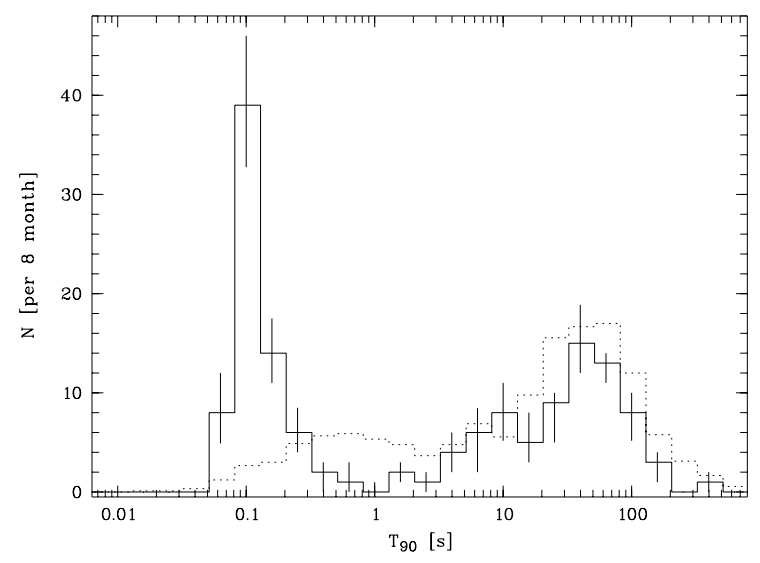

Fig. 3. Distribution of $T_{90}$ for all GRB candidates (solid line) and for 1234 GRBs from the 4th BATSE GRB catalogue (Paciesas et al. 1999; dotted). In order to compare with the SPI-ACS detections, the BATSE distribution is scaled to the elapsed INTEGRAL mission time (8 month). Note the very large fraction of short events compared to BATSE.

be discussed. Other statistical issues (intensity, variability) will be presented in later publications and in Ryde et al. (2003).

Figure 3 shows the distribution of the measure for the duration $T_{90}$ (the time interval starting after $5 \%$ and ending after $95 \%$ of the background subtracted event counts have been observed) for the sample of SPI-ACS GRBs in comparison to the observed distribution of 1234 GRBs from the 4th BATSE GRB catalogue (Paciesas et al. 1999). Despite the small sample, a bimodality in the distribution comparable to that found by BATSE is observed. But two main differences emerge: i) the SPI-ACS sample contains a significantly higher fraction of short burst candidates and ii) the maximum of the short distribution is offset towards shorter duration for the SPI-ACS sample.

The fraction of short $(<1 \mathrm{~s})$ duration GRBs is $\sim 0.48$ (70/145) for the SPI-ACS sample compared to 0.20 for BATSE (Paciesas et al. 1999). As BATSE was observing a softer energy band (50-320 keV) and was therefore more sensitive to X-ray rich (long) GRBs than SPI-ACS, a larger short/long ratio could have been expected for our sample. What is remarkable is

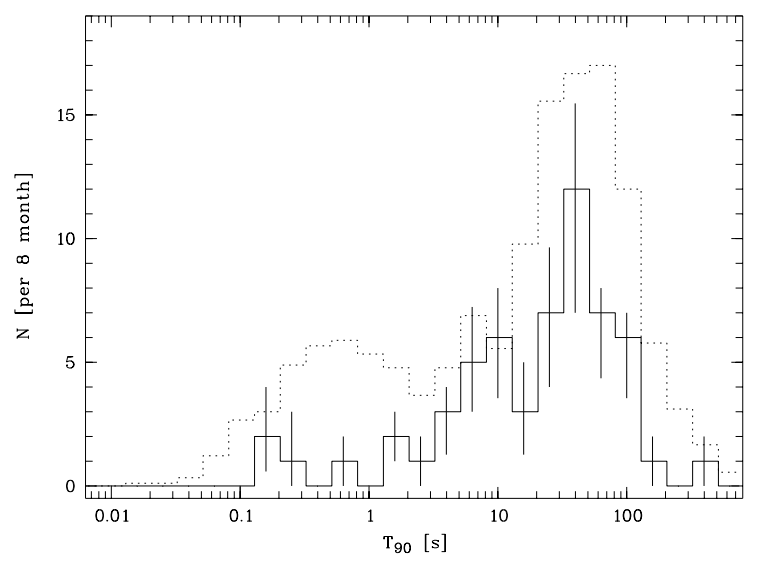

Fig. 4. Same as Fig. 3 except that here only the confirmed SPI-ACS burst sample (solid line) is displayed. While most of the long duration bursts are confirmed (see Fig. 3) for comparison an obvious lack of short GRBs can be noticed.

the sharpness of the short distribution around $0.1 \mathrm{~s}$. Due to the limited time resolution of $50 \mathrm{~ms}$ the short end cannot be sufficiently defined and resolved by our data. BATSE was able to trace the distribution to smaller values of $T_{90}$ due to the better time resolution of $200 \mu \mathrm{s}$.

The offset of the maximum for the short events to smaller $T_{90}$ might be due to the different energy bands of SPIACS and BATSE. An apparently shorter duration is measured as it would be if the bursts would have been observed by BATSE. As $T_{90}$ depends strongly on the instrumental characteristics and as it is still unclear how this measure connects to the source frame quantity for a given burst, the discrepancies are neither surprising nor do they necessarily trace different burst populations.

Still, the connection of the short events with real GRBs is not clear as this population is only marginally observed by other instruments as shown in Fig. 4. While a large fraction $(73 \% ; 55 / 75)$ of the long bursts are confirmed less than $6 \%$ $(4 / 70)$ of the short events were observed by other missions. This might be explained at least twofold. On the one hand we might observe a "real" short and very hard GRB population, 
which could so far only be detected with SPI-ACS due to its high sensitivity at very high energies. As the current IPN members and HETE-II are generally more sensitive at lower energies a high fraction of un-confirmed short (and possible hard) events would not be surprising. These bursts should then have peak energies above $400 \mathrm{keV}$. On the other hand, a significant contribution to these short events from instrumental effects and/or cosmic ray events can not be ruled out. A small contribution might also arise from soft gamma-ray repeaters (SGRs). SGRs are a small class of objects that are characterised by brief and intense bursts of hard X-rays and soft gamma rays. Without localisation SGR bursts can not be distinguished from short GRBs within SPI-ACS. The issue of origin of the short events is certainly of high interest and needs a more detailed investigation

\section{Conclusions}

SPI confirmed so far all six GRBs detected by IBIS within the FoV. With the exception of one GRB SPI was able to derive a GRB position, and for GRBs observed with SPI operating in full telemetry mode, spectra could be extracted. In one case (GRB 030227) some evidence for a hard-to-soft spectral evolution was found in both, ISGRI and SPI

The ACS of SPI has detected 145 GRBs during the first 8 months. At first preliminary analysis of their duration reveals significant differences of their distribution with the one of BATSE. The ACS seems to detect very short GRBs (i.e. shorter than $0.2 \mathrm{~s}$ ). These bursts are much shorter than the typical short burst, which last for $\sim 1 \mathrm{~s}$. It is unclear if this new "population" is real or an artifact of the ACS caused by an electrical effect. Also not understood is the deficiency of the normal short $(<2 \mathrm{~s})$ GRBs. It may be due to the fact that the energy threshold of the ACS is somewhat higher than the one of BATSE. It is the hope that with the continuing observations of GRBs with INTEGRAL these discrepancies can be explained.

Acknowledgements. The SPI project has been completed under the responsibility and leadership of CNES. We are grateful to ASI, CEA, CNES, DLR, ESA, INTA, NASA and OSTC for support. The SPI/ACS project is supported by the German "Ministerium für Bildung und Forschung" through DLR grant 50.OG.9503.0.

\section{References}

Beckmann, V. 2002, Proc. XXII Moriond Astrop. Meeting, p. 417 [astro-ph/0206506]

Beckmann, V., Borkowski, J., Courvoisier, T. J.-L., et al. 2003, A\&A, 411, L327
Boella, G., Butler, R. C., \& Perola, G. C. 1997, A\&AS, 122, 299

Coburn, W., \& Boggs, S. E. 2003, Nature 423, 415

Costa, E., Frontera, F., Heise, J., et al. 1997, Nature, 387, 783

Fishman, G. J., Meegan, C. A., Wilson, R. B., et al. 1989, BATSE: The Burst and Transient Source Experiment on the Gamma Ray Observatory, in Proc. GRO Science Workshop, GSFC, 2

Fishman, G. J., \& Meegan, C. A. 1995, ARA\&A, 33, 415

Galama, T. J., Vreeswijk, P. M., van Paradijs, J., et al. 1998, Nature, 395,670

Götz, D., Borkowski, J., \& Mereghetti, S. 2003a, GCN, 1895

Götz, D., Mereghetti, S., Hurley, K., et al. 2003b, A\&A, 409, 831

Granot, J. 2003, ApJL, submitted [astro-ph/0306322]

Greiner, J., Peimbert, M., Estaban, C., et al. 2003, GCN, 2020

Hjorth, J., Sollerman, J., Møller, P., et al. 2003, Nature, 423, 847

Hurley, K. 1997, in Proc. 2nd INTEGRAL Workshop, ESA SP, 382, 491

Hurley, K., Mazets, E., Golenetskii, S., et al. 2003a, GCN, 1829

Hurley, K., von Kienlin, A., Lichti, G., et al. 2003b, GCN, 2187

Hurley, K., Cline, T., Smith, D. M., et al. 2003c, GCN, 2281

Klebesadel, R., Strong, I., \& Olsen, R. 1973, ApJ, 182, L85

Lichti, G. G., Georgii, R., von Kienlin, A., et al. 2000, in Proc. of the Fifth Compton Symp., AIP Conf. Proc., 510, 722

Lin, R. P., Dennis, B. R., Hurford, G. J., et al. 2002, Sol. Phys., 210, 3

Malaguti, G., Bazzano, A., Beckmann, V., et al. 2003, A\&A, 411, L307

Mereghetti, S., Götz, D., Borkowski, J., et al. 2003a, A\&A, 411, L291

Mereghetti, S., Götz, D., Borkowski, J., et al. 2003b, GCN, 2183

Mereghetti, S., Götz, D., Tiengo, A., et al. 2003c, ApJL, accepted

Mereghetti, S., Götz, D., Beckmann, V., et al. 2003d, A\&A, 411, L311

Metzger, M. R., Djogovski, S. G., Kulkarni, S. R., et al. 1997, Nature, 387,879

Paciesas, W. S., Meegan, C. A., Pendleton, G. N., et al. 1999, ApJS, 122,465

Ryde, F., Borgonovo, L., Larsson, S., et al. 2003, A\&A, 411, L331

Skinner, G. K., Connell, P. H., Naya, J. E., et al. 1997, in Proc. 2nd INTEGRAL Workshop, ESA SP 382, 487

Skinner, G. K., \& Connell, P. H. 2003, A\&A, 411, L123

Stanek, K. Z., Matheson, T., Garnavich, P. M., et al. 2003, ApJ, 591, L17

Ubertini, P., Lebrun, F., Di Cocco, G., et al. 2003, A\&A, 411, L131

van Paradijs, J., Groot, P. J., Galama, T., et al. 1997, Nature, 386, 686

Vedrenne, G., Roques, J.-P., Schönfelder, V., et al. 2003, A\&A, 411, L63

von Kienlin, A., Arend, N., \& Lichti, G. G. 2001, in Proc. of the International GRB workshop held in Rome (Springer), 427

von Kienlin, A., Arend, N., Lichti, G. G., et al. 2003a, in SPIE Conf. Proc. 4851, X-ray and Gamma-ray Telescopes and Instruments for Astronomy, 1336

von Kienlin, A., Beckmann, V., Covino, S., et al. 2003b, A\&A, 411, L321

von Kienlin, A., Arend, N., \& Lichti, G. G., et al. 2003c, in Proc. of the International GRB workshop held in Rome, to be published 